\title{
Erratum to: Radiological findings in an ancient Iranian salt mummy (Chehrābād ca. 410-350 BC)
}

\author{
Lena M. Öhrström ${ }^{1,5} \cdot$ Roger Seiler $^{1} \cdot$ Thomas Böni $^{1,2} \cdot$ Abolfazl Aali $^{3}$ • \\ Thomas Stöllner $^{4} \cdot$ Frank J. Rühli ${ }^{1}$
}

Published online: 28 December 2015

(C) ISS 2015

Erratum to: Skeletal Radiol (2015) 44:811-821
DOI 10.1007/s00256-015-2103-y

"Radiological findings in an ancient Iranian salt mummy" published in Skeletal Radiology June 2015 Vol 44 Issue $6 \mathrm{p}$ 811-821 included a significant amount of material that had previously been published in Metalla, the Journal of the German Mining Museum, Nr.21/2014, 103-112. We take duplicate publication very seriously. This duplication was brought to our attention by one of the authors and we are convinced that it was an honest error. Since Metalla is not widely indexed, and since there is no overlap of the readership, we have obtained agreement from the editor of Metalla to permit publication of this erratum rather than retracting the article.

The online version of the original article can be found at doi:10.1007/ s00256-015-2103-y.

Frank J. Rühli

frank.ruehli@iem.uzh.ch

1 Swiss Mummy Project, Institute of Evolutionary Medicine, University of Zurich, Winterthurerstrasse 190,

8057 Zurich, Switzerland

2 Orthopedic University Hospital Balgrist, Forschstrasse 340, 8008 Zurich, Switzerland

3 Archaeological Museum of Zanjān, Emaarate Zolfaghari, Taleghani Street, Zanjān, Iran

4 Institut für Archäologische Wissenschaften, Ruhr Universität Bochum, Am Bergbaumuseum 31, 44791 Bochum, Germany

5 Present address: Institute of Diagnostic Radiology, University Hospital Zurich, Raemistrasse 100, Zurich, Switzerland 